ENSAYOS

\title{
REFLEXIONES Y DEFINICIONES DESDE LA TEORIA BIOLOGICA DEL CONOCIMIENTO: APRENDIZAJE Y COMPETENCIA EN LA UNIVERSIDAD ACTUAL
}

\author{
Reflections and definitions from the biologic theory of knowledge: learning and \\ competence at the university nowadays
}

\author{
Guido Ruiz Barría \\ Universidad Austral de Chile, Instituto de Farmacia, Campus Isla Teja \\ Casilla 567, Valdivia, Chile. guidoruiz@uach.cl
}

\begin{abstract}
Resumen
En el presente ensayo se evidencia la problemática relacionada con la inexistencia de una definición operativa del término "competencia" en el idioma español, pese a su creciente uso en entornos universitarios y en planificación educacional. Se plantea además que la perspectiva informacionista, desde la cual se explican los procesos de aprendizaje, es incompatible con una definición operativa de competencia. Como alternativa, se propone una perspectiva constructivista basada en la Teoría Biológica del Conocimiento. Se analiza la proyección que tiene esta teoría sobre el proceso de aprendizaje universitario y se propone una definición de competencia desde el constructivismo y considerando las características semánticas otorgadas a dicho término en el lenguaje. Se discuten finalmente las posibles consecuencias derivadas del explicar y definir aprendizaje y competencia desde el constructivismo biológico y se muestra la utilidad de este ejercicio epistemológico para abordar las problemáticas educacionales actuales.
\end{abstract}

Palabras clave: competencia, aprendizaje, autopoyesis, constructivismo, universidad.

\begin{abstract}
In the present essay, the problems related with the inexistence of a clear operative definition of the term "competence" in Spanish language are evidenced, considering the increase in the use of this term in academic environments and educational planning. The author state that the informationistic point of view, commonly used to explain the learning processes, is incompatible with the construction of an operative definition of competence. As an alternative, a constructivistic approach, raised on the Biological Theory of Knowledge is proposed. The projection of this theory over the learning process and its explication is analysed, and a constructivistic definition of competency is proposed, considering the semantic features of the term in the language. Finally, the possible consequences of these constructivistic explanations are briefly discussed, and the useful of this epistemological exercise in approaching some current educational problems is evidenced.
\end{abstract}

Key words: competence, learning, autopoiesis, constructivism, university. 


\section{INTRODUCCION}

\section{DE LA NECESIDAD Y DE LA AUSENCIA DE UNA DEFINICION OPERATIVA DEL TERMINO COMPETENCIA}

Hoy en día y en buena parte del mundo, el alcanzar "competencia" se alza como un objetivo fundamental de los estudios universitarios. De hecho, el contribuir a desarrollar adecuadamente las llamadas "competencias profesionales" en los estudiantes está en el centro de la actual reforma de los currículos y metodologías de enseñanza-aprendizaje en enseñanza superior que se enmarcan dentro del Proyecto Tuning (González y Wagenaar 2002), propuesto y actualmente en desarrollo en Europa y Latinoamérica. Por otro lado, desde hace ya más de una década que se ha venido generando un vasto cuerpo de literatura docente, destinada tanto a profesionales como a académicos, que considera la "competencia" y el desarrollo de ésta como tema central. La recurrencia en la utilización del término "competencia", tanto dentro como fuera de las universidades, parece indicar que en nuestro lenguaje dicho término encierra un concepto de significado conocido, consensuado y que permite la coordinación entre individuos, esto es, que es un término operativo en el lenguaje. Según el Diccionario de la Real Academia de la Lengua Española, al hablar de desarrollo de "competencia", hemos de entender el término competencia bajo sus acepciones de "idoneidad" o "aptitud", además de la de "incumbencia" (Real Academia Española 2001). A mi juicio, y dado lo subjetivo de los términos en los que esta descripción se basa, la definición de la Real Academia, más que una explicación de significado, presenta una orientación útil que, sin embargo, no permite objetivar el concepto de "competencia". Esta dificultad parece estar también presente en otros idiomas como el inglés o el francés en los que el término competencia se asume tiene una traducción directa. Tal vez por esta dificultad, muchos autores, tanto de lengua española como inglesa o francesa, han considerado que el término "competencia" (o su traducción directa) no es un término bien acotado, y otros tantos han discutido y propuesto sendas definiciones para tal. El francés Guy LeBoterf, uno de los autores que más ha abordado el tema de la competencia profesional, señaló hace ya más de una década que este concepto, más que uno operativo, es uno en vías de fabricación (LeBoterf 1994) y que "la dificultad de definirlo crece con la necesidad de usarlo".

Tal como ocurre con otros términos cuya definición operativa resulta problemática (como por ejemplo, los términos "inteligencia", "justicia" o "realidad"), es claro que bajo el término "competencia" existe un concepto cuyo significado es posible intuir, así como es posible para cada persona establecer intuitivamente y bajo su propia perspectiva en qué momento un profesional está actuando de un modo "competente". Coloquialmente decimos que un profesional competente es "aquel que hace bien su trabajo", pero esta idea, fácil de comprender y asumir de un modo individual y subjetivo, es muy difícil de llevar a consenso y objetivar para volverla socialmente operativa. Así, y como ya he señalado, se han propuesto muchas y diversas definiciones de competencia y podría decirse que cada uno de los autores que se han involucrado en el tema en algún momento ha optado por definir "competencia" o "competencia profesional", para luego proceder con sus ensayos. Sostengo que la tendencia general de estas definiciones no es pretender expresar el significado de lo que intuitivamente la sociedad conoce como "competencia", sino describir lo que cada autor entiende como competencia profesional 
o, más aún, lo que a su juicio éste habría de entenderse como "competencia", sobre todo en ambientes universitarios. Es decir, considero que las definiciones propuestas suelen tener un ánimo estipulativo más que descriptivo. Sin ir más lejos, el esquema canónico de las definiciones de competencia profesional suele ser el siguiente:

"Competencia profesional es... -a continuación se enumeran diversas características o factores (como habilidades, destrezas, conocimientos, sentido común, voluntad, etc.) que varían en tipo y número según cada autor- ...que al concurrir en un profesional, garantizan la operatividad laboral de éste, de un modo apropiado y en un contexto laboral real".

La mayoría de las definiciones propuestas están preocupadas de la causa final de la competencia, que por lo general es expresada como la "correcta o adecuada operatividad laboral de un profesional". Esto trae consigo la necesidad de conocer entonces qué es un "modo correcto" de actuar u operar. Por otro lado, en busca de un significado potencial del término, muchas de las definiciones se remiten a consideraciones etimológicas o saltan bruscamente a consideraciones psicopedagógicas, en lugar de basarse en las propiedades semánticas que se descubren en el uso habitual de un término tan común en nuestro lenguaje. Por todo ello, estas definiciones en su mayoría no colaboran a solucionar el problema de operatividad en el lenguaje para el término "competencia".

La ausencia de una definición operativa de competencia profesional y el súbito interés y necesidad de construirla plantea, iniciado el siglo XXI, una paradoja para la universidad como institución. Esto, si pensamos que la universidad históricamente, y sobre todo tras la revolución industrial, ha tenido dentro de sus objetivos el formar especialistas en diversas disciplinas destinados a ejercer un conjunto de labores indispensables para la organización y el funcionamiento de la sociedad. A la luz de estos objetivos, es fundamental que la universidad maneje una definición de "competencia" acorde con el uso real del término en el lenguaje, pues esto muy probablemente le facilitará la tarea de generar profesionales competentes. Nótese que, dada la ausencia de una definición operativa, las universidades y los docentes suelen basarse en un concepto intuitivo y personal de lo que es competencia, sin preocuparse demasiado por establecer un consenso al respecto. Llevado a una analogía, esto es como si cada trabajador de una fábrica aportara de modo libre para producir lo que él intuye que es el factor $\mathrm{C}$, que no necesariamente se asemeja a lo que intuye otro operario o, lo que es más importante, el consumidor del factor $\mathrm{C}$, en este caso la sociedad toda. Esto deja en evidencia el gran problema derivado de la falta de consenso efectivo respecto al significado del término "competencia".

\section{DE LA INCOMPATIBILIDAD ENTRE LA PERSPECTIVA INFORMACIONISTA DE ENSEÑANZA- APRENDIZAJE Y LAS DEFINICIONES EXISTENTES DE COMPETENCIA PROFESIONAL}

Llama fuertemente la atención el que las teorías de aprendizaje de relevancia actual tengan todas una mayor o menor orientación constructivista. No obstante, en el uso cotidiano del lenguaje, e incluso en ambientes universitarios, es aún muy común que oigamos hablar de "entregar conocimiento", de "entregar información", "transmitir información", o de que el estudiante "tome" o "haga suyo" el conocimiento. Esto evidencia que aún la sociedad, y dentro de ella los docentes, asume la premisa informacionista de que el humano es un sistema al que se le puede ingresar información o conocimiento externamente determinado. 
Mi planteamiento es que desde una perspectiva diferente, no informacionista y radicalmente constructivista, es posible explicar el proceso de formación profesional, y que desde esta perspectiva se facilita la construcción de una definición operativa de lo que intuitivamente se conoce como competencia y, por ende, el abordaje de la problemática de formar profesionales competentes en la universidad.

La perspectiva de la enseñanza universitaria occidental tradicionalmente se ha basado en la concepción de que la información es un "algo" transmisible, un input sobre el estudiante, que determina cambios en la conducta futura de éste. Así, este proceso de transmisión de la información o conocimiento comúnmente consiste en la descripción, por parte del docente, de un cuerpo ordenado de realidades objetivas y validadas que el estudiante no distinguía sino hasta el momento en que es ilustrado por el educador, quien "le abre los ojos" a un nuevo segmento de la realidad. Estos nuevos segmentos por lo general se constituyen de definiciones de conceptos, de taxonomías y de jerarquizaciones. Es decir, son redes de conceptos. En mi opinión, el proceso de formación profesional explicado desde la perspectiva de la transmisión de la información es difícilmente compatible con lo que intuitivamente se conoce como competencia, es incompatible con muchas de las definiciones actuales de competencia, dificulta la definición operativa de competencia y no permite establecer un modelo educativo atento al desarrollo de lo que se intuye como competencia. Para justificar mis aprehensiones, propongo el siguiente análisis:

Si nos orientamos por las definiciones existentes, y entendemos competencia como un conjunto de factores diversos que al concurrir en un individuo garantizan cierta operatividad, y luego nos situamos en la perspectiva informacionista, veremos que para que un estudiante llegue a demostrar cierta operatividad, sea cual fuere, se han de cumplir las siguientes premisas:

a) La información transmitida es siempre la adecuada para contribuir a generar la operatividad (es causal).

b) La transmisión de información se realiza siempre de un modo eficaz y completo.

c) Los estudiantes son igualmente desconocedores de las nuevas realidades al momento de recibir la información.

d) Existe una funcionalidad cognitiva idéntica en todos los estudiantes, de modo que el procesamiento de la información se realiza de un modo idéntico en cada uno de ellos, para dar origen a una operatividad o competencia idéntica.

Estas premisas se revelan como equivocadamente reduccionistas y sencillamente falsas, sobre todo en lo que respecta a los estudiantes en su naturaleza humana, puesto que para que se cumplan es necesario asumir de ellos una variabilidad estrechísima. Biológicamente, esto equivaldría a decir que cada estudiante es una réplica del otro momento a momento, durante el transcurrir de su formación universitaria. Además, frente a un concepto multidimensional de competencia profesional, la perspectiva informacionista se vuelve aún menos útil, al ser muy difícil o sencillamente imposible modular mediante la llamada "transmisión de información" las dimensiones afectivo-emocionales e incluso las técnicas del estudiante. 


\section{UNA TEORIA BIOLOGICA COMO PERSPECTIVA DE EXPLICACION DEL PROCESO ENSEÑANZA-APRENDIZAJE EN LA UNIVERSIDAD}

La teoría que propongo como perspectiva explicar el proceso de enseñanza-aprendizaje en la universidad y para definir competencia profesional parte desde la explicación del concepto de vida. Fue desarrollada por los biólogos chilenos Humberto Maturana y Francisco Varela y se le conoce como Teoría de la Autopoyesis. Esta teoría analiza el surgimiento fenómeno vital (lo vivo) y de la conciencia y el lenguaje (lo humano) partiendo exclusivamente desde la biología. Así, la teoría discute las raíces biológicas del conocimiento humano, excluyendo absolutamente la idea común de transmisión de información como input, por ser ésta incompatible con una explicación biológica del ser. Dada su implicación con los fenómenos del ser y el conocer, también se conoce a esta teoría como Teoría Biológica del Conocimiento.

La Teoría de la Autopoyesis, formulada y propuesta a comienzos de la década de los 70 (Maturana y Varela 1973), ha tenido gran impacto en diversas áreas del conocimiento, incluyendo la sociología y legislación (Nelken y Priban 2001; Luhmann 1982), la administración de negocios (Mingers 1995) y la psicoterapia (Snyder 1999). Es además el paradigma de la cibernética de segundo orden (Zeleny y Hufford 1992). En el área biológica, su repercusión ha sido más lenta, aunque no por ello menor. A mediados de la década pasada eminentes biólogos como Lynn Margulis aceptaron el concepto de autopoyesis como parte integral de la descripción de la vida (Fleischaker y Margulis 1986; Margulis and Sagan 1995), y los últimos años la aceptación, el impacto y la divulgación de la Teoría Biológica del Conocimiento ha crecido sostenidamente dentro de la comunidad científica biológica. Actualmente conceptos propios de la teoría, como autopoiesis o clausura organizacional sirven de base tanto a discusiones teóricas respecto al fenómeno vital (Letelier et al. 2003; McMullin 2000; Stewart 2000; Scott 2000) como al diseño de modelos de vida artificial en el laboratorio (Mavelli y Luisi 1996; Szostak et al. 2001; Deamer et al. 2002). Por mi parte, recientemente he abordado la explicación del fenómeno de dolor neuropático desde dicha perspectiva (Ruiz 2005) (manuscrito in extenso en preparación).

\section{OBJETIVOS}

Los objetivos de este ensayo son: 1) Proponer una definición potencialmente operativa del término "competencia", explicando los procesos de enseñanza universitaria y formación profesional desde una perspectiva no informacionista basada en la Teoría Biológica del Conocimiento y, en este proceso, 2) analizar brevemente las implicancias de estas explicaciones para la formación universitaria actual.

\section{PLANTEAMIENTOS BASICOS DE LA TEORIA BIOLOGICA DEL CONOCIMIENTO}

Comenzaré por presentar, de un modo somero, algunos aspectos de la Teoría Biológica del Conocimiento. Sólo me limitaré a exponer los conceptos fundamentales que estimo facilitarán el desarrollo de un lenguaje básico, el cual es necesario para lograr 
coordinación en la reflexión motivada por el ensayo. Aclaro que, tanto los enunciados de la teoría como las reflexiones que los autores de ésta han hecho respecto a sus implicaciones, se suceden de una referencia bibliográfica. Las descripciones, reflexiones y ejemplos dados para cada enunciado no acompañadas de referencia, corresponden al autor de este ensayo.

1. Ser vivo y autopoyesis. Aquello que distingue a los seres vivos del ambiente no vivo no es una lista de características específicas de los seres vivos, sino la organización de éstos. Dicha organización es, pues, la que define a los seres vivos. Los seres vivos son aquellos sistemas que transcurren con conservación de su identidad organizacional, que es una organización autopoyética (Varela et al. 1974). Como autopoyesis (del griego $\alpha \cup \tau o \zeta=$ por sí mismo; $\pi$ otعıv=hacer) se define aquella organización que se genera a sí misma, en cuanto a sus procesos y a sus constituyentes, en forma autonómica y con clausura operacional respecto al ambiente (Varela et al. 1974; Varela 1979).

Aunque la discusión acabada de este vasto concepto se aleja de las pretensiones de este ensayo, creo importante especificar que el concepto de clausura operacional no implica hermetismo de los seres vivos hacia el ambiente. Lo que sostiene es que es únicamente la dinámica estructural y organizacional de los seres vivos la que determina qué factores del ambiente participarán de sus procesos.

2. Clausura y determinismo organizacional de los seres vivos. La definición anteriormente propuesta se hace cargo del determinismo estructural de los seres vivos, es decir, que todo cambio que experimente un ser vivo, aunque gatillado por el ambiente, está determinado por la dinámica propia del ser vivo. El ambiente, entonces, no determina ni condiciona ni estipula qué cambios experimentará un ser vivo, sólo los promueve (Maturana y Varela 1987). Este aspecto es fundamental para la comprensión de la teoría. A modo de ejemplo, un flujo de fotones no producirá el mismo efecto sobre una piedra o sobre la hoja de una planta o sobre la piel de un humano o sobre la pupila de un gato. No es entonces el ambiente el que condiciona los cambios observables en un sistema distinto, sino la estructura del sistema y, en caso de los sistemas vivos, la dinámica estructural de éstos.

3. Acoplamiento estructural. El ser vivo y el ambiente, que incluye otros seres vivos, están en constante interacción. El ambiente gatilla diversos cambios en el organismo, cambios denominados perturbaciones. A la sucesión de perturbaciones que no resultan directamente en la pérdida de la organización del ser vivo, sino en cambios de su dinámica estructural con conservación de la organización, Maturana y Varela la denominan acoplamiento estructural entre ser vivo y ambiente (Varela et al. 1974).

4. Clausura operacional del "testigo" y generación de la realidad individual. La cultura occidental, proyectando la filosofía aristotélica, ha tratado la realidad sensorial como si fuese algo externo e independiente del ser vivo, y ha considerado la información como algo transmisible. Según los planteamientos biológicos anteriormente descritos, lo que un humano conoce por realidad sensorial no es algo objetivo que esté definido exteriormente y que uno recoja como información externa. Así, los cambios en la dinámica estructural del sistema nervioso producen cambios en la realidad individual. Luego, 
dados los sistemas nerviosos de un gato y un humano, con sus respectivas dinámicas estructurales distintas, las realidades que gato y humano conocen son distintas. Lo que se establece de este modo es que no hay una realidad objetiva, sino tantas realidades como "testigos" y que en los seres vivos el proceso de transmisión de información como tal no existe. A la tendencia a describir perturbaciones como si éstas estuviesen determinadas externamente, Maturana la denomina la "falacia descriptiva" (Maturana 1975). Nótese que para los seres vivos, ninguna información o conocimiento proviene del exterior, cada ser vivo conoce la realidad desde la profunda soledad de su clausura operacional (Maturana 1978; Maturana y Varela 1987).

5. Conducta. A la descripción de los cambios en la dinámica estructural de un ser vivo, realizada por un testigo ${ }^{1}$ externo, se le denomina conducta (Maturana y Varela 1987). Nótese que esta definición implica que no existe una conducta absoluta. La conducta, como descripción, es determinada por la dinámica estructural del testigo, en cuanto a lo que éste puede conocer y describir. El concepto de testigo u observador de la conducta es un factor muy importante a la hora de comprender nuestras interacciones en el lenguaje, puesto que "todo lo dicho, es dicho por alguien" (Maturana y Varela 1987), y todo concepto que lleve implícita la observación de un ser vivo a otro, necesariamente ha de ser definido mencionando al testigo dentro de la definición. Sostengo que esto es muy importante si se pretenden construir definiciones operativas en el lenguaje sobre términos que encierran conceptos intuitivos y subjetivos.

Asumo que es imposible hacerse una idea global e inmediata de la teoría a partir de los pocos enunciados expuestos. Por ello, invito a los lectores a profundizar en las obras de Maturana, Varela y otros, referidas a lo largo del presente ensayo.

\section{EL PROCESO UNIVERSITARIO EXPLICADO DESDE LA PERSPECTIVA DE LA TEORIA BIOLOGICA DEL CONOCIMIENTO}

1. Definición del término aprendizaje. Los seres vivos están en constante interacción con el ambiente y con otros seres vivos, y se acoplan estructuralmente al ambiente y a los seres vivos con los que interactúan recurrentemente, coordinando sus ontogenias. Estas interacciones constituyen el dominio de interacciones del ser vivo. Dado un ser vivo expuesto a un testigo, el acoplamiento estructural del ser vivo y ambiente generará conductas a describir por el testigo. Estas conductas constituyen el dominio conductual del ser vivo. El dominio de interacciones y el dominio conductual son dominios asociados pero son dominios disjuntos ante un testigo. No es posible para un testigo predecir o aventurar con certeza cuál interacción con el ambiente estuvo o está relacionada causalmente y hasta qué punto con una conducta observada. Esto, dado que una interacción se relaciona con las conductas a través y durante toda la ontogenia del ser

1 Maturana y Varela denominan al que describe la conducta, observador. Yo prefiero el término testigo, pues no da preferencia a ningún sentido sobre otro. Por otro lado, el término testigo lleva implícito el hecho de describir, dado que testigo es a la vez "quien da testimonio" como quien "presencia o adquiere directo conocimiento de una cosa". 
vivo, por lo que una conducta presente está relacionada con la biofísica presente y por ende con toda la ontogenia del ser vivo. Por ello, lo que un testigo denomina conducta innata o instintiva, es aquella conducta para la cual el testigo no puede asociar ninguna relación con interacciones con el ambiente de un modo fundamental y que, en la falacia descriptiva, puedan parecerle determinantes. Para ilustrar este concepto propongo el siguiente ejemplo: es conocido que ciertos mamíferos superiores, al ser criados en cautiverio pueden perder o modificar muchas de sus conductas instintivas, incluidas las conductas reproductivas. Así y todo, muchas especies logran reproducirse pero modificando en ocasiones dramáticamente su conducta. Esto deja en evidencia que la conducta instintiva o innata de los animales se debe a los cambios de su dinámica interna producto de la historia de interacciones con el ambiente. Si el ambiente cambia, las conductas llamadas instintivas también. Lo que ocurre es que un testigo humano no es capaz de reconocer a priori qué factores del ambiente del animal cambiaron y supusieron un cambio en la conducta animal observable y describible por el humano (testigo). Por el contrario, lo que un testigo denomina conductas aprendidas, son conductas para las cuales el testigo puede distinguir fácilmente relaciones con interacciones previas de un ser vivo con el ambiente, asociando interacción y conducta de un modo unívoco del tipo causa-consecuencia. En las conductas aprendidas tiene especial importancia el lenguaje como interacción con el ambiente. En la falacia descriptiva, el lenguaje aparece como un instrumento de comunicación, pero explicando la comunicación como la transmisión de información, en la que la información es un determinante externo de conductas del ser vivo. Según la Teoría Biológica del Conocimiento, el lenguaje se genera en, y por el acoplamiento estructural de, dos seres lenguajeantes y sólo consigue la coordinación de éstos. Dado que el ambiente del ser vivo está en constante cambio, producto de sus interacciones con todos los seres vivos, ambiente y ser vivo transcurren a la deriva, o en una deriva ontogénica (Maturana 1982). Dicha deriva no tiene más causa final que el mantenimiento de sí misma, como acoplamiento estructural constante entre seres vivos y ambiente. El ser vivo se acopla estructuralmente al ambiente del modo que su dinámica estructural presente determina. Esto implica que, según cada dinámica estructural, habrá una manera diferente de acoplarse estructuralmente al ambiente. Para el ser vivo como unidad autopoyética no hay maneras mejores o peores de acoplarse al ambiente, todo acoplamiento es correcto en cuanto no implica destrucción del ser vivo. Por esto, para la Biología del Conocimiento el aprender es la consecuencia inequívoca de la ontogenia de un ser vivo que cambia en forma congruente con el ambiente. En palabras de Maturana: "no hay diferencia intrínseca entre conducta instintiva y conducta aprendida, ya que ambas son el resultado de la epigénesis del organismo y surgen en cada caso, como consecuencia inevitable de la historia de interacciones de éste con conservación de la organización..." (Maturana 1982).

En base a la discusión anterior, la definición que propongo para lo que denominamos aprendizaje, desde la Teoría Biológica del Conocimiento y basándome estrictamente en el uso de los términos en el lenguaje, es la siguiente:

Dados un ser vivo, un testigo y un ambiente A del cual el testigo conoce ciertas variables:

Aprendizaje es la ampliación de los dominios conductual y de interacciones en el lenguaje de un ser vivo, tras un tiempo $(\Delta t)$ de interacción con A y que el testigo 
considera: a) condicionante de operatividad y b) congruente con las variables de A que él conoce y con el número de interacciones entre ser vivo y A que estima que se pueden producir en $\Delta \mathrm{t}$.

A mi modo de ver, esta definición incluye todos los factores que se intuyen o conocen del término aprendizaje en nuestro lenguaje:

- El evaluador, como testigo, puede ser el mismo ser vivo que ha aprendido o está aprendiendo, pero en un tiempo futuro: "En ese entonces yo aprendí...".

- No es siempre necesario que el ser vivo conozca que está aprendiendo o que está siendo sometido a un proceso de aprendizaje. Eso puede decidirlo el testigo tras un contraste retrospectivo: "En aquel viaje, él aprendió que...".

- El incremento del dominio conductual o del lenguaje debe ser condicionante de operatividad. Así, en el lenguaje describimos el aprendizaje en función de la operatividad que condiciona: "Ella ha aprendido a interpretar un análisis clínico, él $\underline{a}$ lanzar la jabalina, ellos a robar, él a guardar silencio”.

- El aprendizaje se constata como una ampliación de los dominios conductuales y del lenguaje, es decir, un evaluador sólo puede juzgar si hay aprendizaje tras interaccionar o al menos exponerse al ser vivo o su obra: "No sabré si aprendió hasta que le vea, o lea sus escritos".

- El aprendizaje siempre se da tras un período de exposición a A con variables que el testigo conoce, y que, equivocadamente, considera determinantes absolutas y externas del proceso de aprendizaje. A modo de ejemplo: Si alguien que conocemos aparece súbitamente hablando japonés, sin haber ido a Japón, sin seguir un curso o al menos oír japonés en alguna parte, supondrá, además de una perturbadora sorpresa para nosotros, el que pensemos que esa persona "habla japonés instintivamente (!)". Estos factores (exponerse a un medio donde se hable japonés, seguir un curso de japonés) aparecen como los determinantes en el aprendizaje de un idioma, pero no pueden considerarse causas eficientes, en tanto hay personas que pueden vivir toda su vida en Japón, y no aprender a hablar japonés, porque su biofísica y ontogenia así lo determinaron. Aunque las variables externas están muy relacionadas con el aprendizaje y participan de él, su presencia o interacción no garantiza aprendizaje. Según la lógica inductiva sobre la que se construye la ciencia observacional moderna, las variables externas no pueden ser consideradas causas eficientes en seres vivos.

- El que el aprendizaje se desarrolle en un tiempo $(\Delta \mathrm{t})$ de exposición e interacciones con A (etapas), implica que es un proceso. Observemos que, si el incremento del dominio conductual o de lenguaje es muy elevado y $\Delta \mathrm{t}$ demasiado estrecho para permitir las interacciones con el ambiente que nosotros, como evaluadores, estimamos necesarias, de inmediato dudamos sobre si el sujeto ha aprendido algo muy rápidamente o simplemente ya lo sabía. Por ejemplo, si tras sólo una clase de mecánica automotriz un sujeto comienza a reparar coches afanosamente, dudaremos de su eficiencia como reparador. Si comprobamos que es eficiente, diremos del sujeto "él no ha aprendido a reparar coches en una sola clase, esto lo sabía desde antes".

2. Breve explicación del proceso enseñanza-aprendizaje en la universidad desde una perspectiva no informacionista. Las sociedades humanas se caracterizan, entre otras cosas, por la especificidad de roles de algunos de sus miembros dentro de su organización. 
Durante el transcurrir de la filogenia humana, diversos han sido los modos de asignación de papeles o roles a los individuos que constituyen sus sociedades. En general, dichos modos se han mantenido entre los extremos distinguibles de a) predestinación hereditaria, donde cada individuo desarrolla forzosamente el papel que su progenie o su casta le obliga y b) completa liberalización, en la que cada individuo tiene libertad para elegir y ejercer el papel que estima conveniente. En rigor, en las sociedades posmodernas rara vez se dan estos extremos, pues de una u otra forma siempre existe una situación mixta con tendencias a alguno de los dos.

Cuando la organización de las sociedades humanas es poco compleja, los humanos aspirantes a desarrollar un papel en ella, ya sea de motu proprio u obligados, se acoplan estructuralmente a otros humanos, que les sirven de modelos, y al ambiente de éste o éstos. A partir de ahí, aspirante y modelo coordinan sus ontogenias en función del ambiente laboral, y se someten a constantes interacciones en el lenguaje. A este método, por existir antes de cualquier teoría del conocimiento y del aprendizaje, lo denomino método natural de generación de competencia. En este método, el estudiante es considerado competente una vez que el modelo o la sociedad así lo estiman. A medida que la población y el dominio tecnológico de la sociedad humana aumentaron, la organización de ésta se volvió más compleja, y surgieron las universidades como ambiente modulador de la ontogenia del ser vivo o inductor de aprendizaje. La reflexión sobre la cual la sociedad humana actualmente sistematiza la enseñanza universitaria se plantea sobre dos axiomas incompatibles con la definición operativa de ser vivo:

1. La información es algo externo que se transmite, y la causa final del lenguaje no es producir el acoplamiento estructural entre humanos, sino transmitirse información. La información o conocimientos que se entregan condicionan el operar futuro de un estudiante humano.

2. Las variables ambientales determinan o definen la ontogenia de los estudiantes humanos. Es decir, todos los individuos forzosamente han de responder del mismo modo ante las variables ambientales que se conocen o controlan. El estudiante que no responde del modo esperado es anómalo y, por lo tanto, reprobable.

Basándose en estos axiomas, la universidad se explica como el espacio físico donde se produce la transmisión de conocimiento. Por el contrario, desde una perspectiva no informacionista, los estudiantes, los profesores y la universidad han de explicarse de otro modo. A continuación propongo las siguientes explicaciones de los conceptos de estudiante, docente y universidad, desde la perspectiva no informacionista de la Teoría Biológica del Conocimiento.

Los estudiantes son humanos en un estadio de su ontogenia que aspiran a adquirir un papel funcional y productivo dentro de una organización social. Con dicho objeto, el estudiante o aprendiz se somete a ser modulado en su ontogenia por medio de constantes y dirigidas modificaciones en su dominio de interacciones con el ambiente.

Los profesores son moduladores de la ontogenia de los estudiantes. Esta modulación se habría de realizar sin que el docente intentase identificar las variables ambientales que determinarán el aprendizaje en todos y cada uno de los estudiantes. Antes de convertirse en un descriptor-explicador, tarea que en un futuro tan cercano como el presente puede realizar una máquina, el docente habría de lenguajear con los estudiantes, para 
posibilitar el desarrollo de la lógica propia de la disciplina que imparte. Según Maturana "Los humanos razonan según la lógica de su lenguaje, y este lenguajear con otros seres vivos o con ellos mismos al reflexionar modula su ontogenia constantemente y en un proceso circular" (Maturana 1995). Las interacciones en el lenguaje se generan lenguajeando. En gran medida aprender una disciplina es aprender un lenguaje y a lenguajear y reflexionar en él, con la lógica constitutiva de cada disciplina. Si un estudiante interacciona recurrentemente en el lenguaje bioquímico con su docente de bioquímica, y promueve la ampliación de su domino lingüístico (mediante interacciones lingüísticas con el ambiente, como leer bioquímica, escuchar descripciones bioquímicas, resolver ejercicios de bioquímica) aprehenderá a "bioquimizar", es decir, a interpretar, describir y explicar el ambiente y a sí mismo, con la lógica del lenguaje bioquímico de su maestro, pero generando sus propios razonamientos. Es interesante observar que la búsqueda de la generación del lenguaje disciplinario se evidencia cada vez que los estudiantes persiguen interaccionar en el dominio del lenguaje de la disciplina que estudian, discutiendo en grupo el tema que estudian, reflexionando o hablando de ésta. Esto es, generar las interacciones consensuales a partir de otras interacciones consensuales (lenguaje), que permiten establecer una lógica disciplinaria consensuada.

La universidad es el ambiente en el cual se modula la ontogenia de los estudiantes en su fase última de preparación. La universidad, como ambiente, interacciona constantemente con los estudiantes, que a su vez son parte del ambiente de los otros estudiantes. Esto genera una problemática, puesto que un estudiante, en un ambiente de estudiantes y aislado del medio profesional, aprenderá a ser estudiante mas no a ser profesional. Por esto, la universidad y todos quienes pueden administrar de algún modo dicho ambiente han de velar por promover un ambiente de futuros profesionales, con referencias explícitas y constantes al profesional requerido, con docentes que se expliquen a sí mismos como modelos de disciplinas y propicien interacciones recurrentes en el lenguaje y, sobre todo, haciendo lo posible por no considerar constantemente que es posible conocer de qué modo las variables del ambiente condicionan la ontogenia del estudiante, sino sometiendo tempranamente al estudiante al ambiente laboral y/o a problemas, situaciones y entornos que repliquen en todo cuanto sea posible, el medio laboral y su problemática. Conscientes o no de las aproximaciones constructivistas al concepto de aprendizaje, muchas universidades han adoptado durante los últimos años dinámicas que apuntan a lo discutido en este párrafo, y tanto el aprendizaje basado en problemas (problem-based learning) como el temprano contacto con el medio laboral forman parte de las reformas actuales en formación universitaria (Savery 2006).

\section{CONSTRUCCION DE UNA DEFINICION OPERATIVA DE COMPETENCIA DESDE UNA PERSPECTIVA CONSTRUCTIVISTA}

1. ¿QUE ES COMPETENCIA EN NUESTRO LENGUAJE? CARACTERIZACION DEL TERMINO COMPETENCIA EN NUESTRO LENGUAJEAR COTIDIANO. La competencia es una designación de estado o atributo. Tal como la belleza y la simpatía, la competencia es una designación de estado o atributo. Esto lleva implícito la existencia de un testigo quien atribuye o designa. Si el testigo no existe, no existe el atributo. La competencia es entonces un "obsequio" que hace un testigo a otro ser vivo o a él mismo, otorgándole dicha 
designación. Así, decimos que el sujeto A es competente tal como podemos decir que es simpático, si así nos parece. Si un sujeto $\mathrm{C}$ dice de sí que es competente, diremos que se atribuye competencia a sí mismo.

La competencia es sólo atribuible a seres vivos o sus colectivos. Pese a que trabajamos diariamente con escritorios, teléfonos y computadores, jamás diremos, a menos que sea irónicamente, que un teléfono es un incompetente porque se ha estropeado o no cumple nuestra expectativa. Por otro lado, si un perro policial detector de drogas sufre algún problema de olfacción, tal vez diremos que el animal ya no es competente en su labor. Una empresa o asociación, como colectivo de seres vivos, también puede ser calificada como competente o incompetente en nuestro lenguaje.

La calidad de competente la atribuye la sociedad. Como he mencionado, las definiciones operativas de conceptos plenamente subjetivos han de incluir al testigo dentro de su definición. Es decir, se debe inscribir su subjetividad para volverlas objetivas, de lo contrario no pueden ser operativas y se vuelven constantemente fuentes de conflicto y confusión. Esto es lo que he realizado en la definición de aprendizaje y lo que Maturana y Varela realizan en la definición de conducta. En el caso del término competencia, podría estar tentado a declarar que el testigo puede ser cualquier humano. No obstante, la competencia, y sobre todo la competencia laboral, hace referencia a la participación de un ser vivo dentro de una sociedad, realizando un papel que la sociedad requiere para mantener su organización. Un sujeto competente para una sociedad como médico puede ser totalmente incompetente como tal para otra, porque las necesidades de una y de otra son distintas y el papel del médico en cada una de esas organizaciones habrá de ser distinto. Por tal razón, no obstante la calidad de competente puede ser otorgada por cualquier humano hacia otro, en nuestro lenguaje la competencia laboral es una denominación que ha de ser otorgada por la sociedad, o un representante de ésta, validado por ésta.

La competencia se contrasta contra una expectativa. Un testigo o la sociedad otorgan la calidad de competente a un ser vivo en función de lo que se espera o conoce respecto a su papel dentro de la organización de la sociedad. Cuanto mayor es el conocimiento del testigo respecto a dicho papel, los elementos de juicio se amplían. Por ejemplo, si un testigo pregunta acerca de un medicamento a un farmacéutico y éste dice no saber, el testigo no le atribuirá competencia al farmacéutico, toda vez que tenga una expectativa respecto al papel del farmacéutico como descriptor de medicamentos.

La competencia es un atributo único e indivisible. Tanto en ambientes académicos como en textos docentes es cada vez más frecuente oír o leer acerca de "competencias", en plural, y de desarrollo de dichas "competencias". Esta idea, como estrategia, puede ser eficaz al diseñar programas de estudio y no pretendo entrar en una discusión metodológica al respecto. Sin embargo, sostengo que, al menos en el idioma español, la pluralización del término competencia es un ejercicio relativamente reciente, promovido por las reformas educacionales y las teorías que la sustentan. El problema es que la sociedad, para otorgar la calidad de competente, no realiza una evaluación razonada o reflexión en base a operaciones aisladas, o factores de operatividad o habilidades o algún tipo de unidades en los que se pueda fragmentar la calidad de competente. Para 
la sociedad, en el lenguaje cotidiano, un individuo es o no es competente, y hay poco espacio para términos medios. Ocurre, pues, lo mismo que con otros atributos como la belleza, la inteligencia o la simpatía. Suena sencillamente absurdo pensar un curso de "desarrollo de simpatías", o de "desarrollo de inteligencias" o de "desarrollo de sinceridades", atributos únicos e indivisibles como la "competencia". Para la sociedad no existen "competencias", sólo competencia. Esta situación no es trivial, puesto que si la universidad no habla el mismo lenguaje que la sociedad, difícilmente se puede llegar a una comprensión y a un consenso respecto al significado del término "competencia" profesional. A mi juicio, urge un amplio abordaje de este tema, dentro del uso y abuso del término competencia.

La competencia no se puede desarrollar. Un atributo no se puede desarrollar, pues depende sólo del criterio del testigo que lo otorga. Así, si el testigo o el criterio de éste cambian, su juicio respecto al atributo también. En el caso de la competencia, por ser el testigo la sociedad toda, es posible generar un ambiente que module la ontogenia de los estudiantes de modo que se acomode al criterio de lo que la sociedad conoce como profesional competente. Pero esto requiere una universidad e instituciones muy atentas a lo que la sociedad espera de cada profesional, y requiere que los estudiantes sean enfrentados al ambiente laboral presente o, en su defecto, a réplicas de ambientes tales como los que se encontrarán en su labor.

2. ¿EN QUE SE BASA LA SOCIEDAD PARA OTORGAR LA CALIDAD DE COMPETENTE A UNA PERSONA? Como he señalado anteriormente, la sociedad no otorga la calidad de competente en base a un juicio consciente o a una reflexión, ni menos a un listado de factores. Se trata, más bien, de una apreciación o proceso inconsciente o intuitivo. En un momento dado existe una expectativa para la sociedad respecto a la conducta de un profesional, y ésta o se cumple o no se cumple. Sostengo que dicha apreciación se lleva a cabo en base a la observación de todas las interacciones de un ser vivo, tanto en el dominio conductual como del dominio del lenguaje, que se producen en el ambiente laboral. Esta es la razón por la que las definiciones de competencia, propuestas como caracterizaciones, plantean largas listas de factores que aún así no parecen abarcar lo suficiente del concepto competencia. Cuando un individuo es calificado como competente, lo que se califica es toda su ontogenia, observada en su conducta y en sus interacciones actuales en el lenguaje, toda vez que el individuo está acoplado a un ambiente laboral e inmerso en la sociedad calificadora.

Pese a lo anterior, creo que es posible distinguir dos factores muy básicos, a partir de los cuales un sujeto puede optar a ser calificado como competente por la sociedad:

1. Acoplamiento estructural con la sociedad y el ambiente laboral. Para ser calificado como competente, un ser vivo necesariamente debe estar acoplado estructuralmente con la sociedad, y debe participar de ella y de su organización. Cualquier situación que atente contra el acoplamiento estructural de un individuo con la sociedad atentará contra la competencia. Las enfermedades postradoras o infectocontagiosas, los trastornos cognitivos, el alcoholismo, ciertas farmacodependencias, conductas criminales o antisociales, entre otras situaciones, atentan contra la competencia en tanto atentan contra el acoplamiento estructural entre el ser vivo y la sociedad. Por otro lado, un ser vivo debe estar acoplado al ambiente laboral donde ha de trabajar. 
2. Funcionalidad y conservación de la identidad profesional como parte de su ontogenia. $\mathrm{Al}$ trabajar, los humanos asumen un sistema de relaciones y conductas con la sociedad, según las necesidades de ésta, que generan su identidad laboral. Sin embargo, no se trata sólo de realizar una serie de operaciones de forma automática. En tanto ambiente y ser vivo transcurren en una deriva ontogénica y cambian en forma conjunta y congruente, el ser vivo debe conservar su adaptación y mantener la identidad laboral que la sociedad le demanda dada la organización de ésta. Por lo tanto, el dominio conductual que constituye la operatividad debe cambiar de modo de conservar la identidad profesional del ser vivo dentro de la sociedad. Un biólogo debe ser biólogo hoy y mañana y en veinte años más, y su funcionalidad es hacer biología y biologizar. A modo de ejemplo: si la funcionalidad de un constructor de casas es construir casas, y el material con el que se construyen las casas cambia, el constructor deberá modular su operatividad para hacer casas del material nuevo, de lo contrario corre el riesgo de dejar de ser constructor, y ciertamente, en algún momento será designado por la sociedad como un constructor incompetente.

\section{Propuesta de una definicion no teleológica y potencialmente operativa de competen-} cia profesional. En base a lo expuesto y discutido en el presente ensayo, propongo la siguiente definición de competencia:

"Dado un individuo que participa y está socialmente adaptado a un medio laboral, y que pertenece, participa y está adaptado a una sociedad:

Competencia es un atributo otorgado al individuo por la sociedad. Dicho atributo se otorga tras considerar todas las interacciones del individuo con su medio laboral material y humano, y toda vez que el individuo cumple las expectativas que la sociedad se genera en cuanto a funcionalidad con conservación de su identidad laboral en el tiempo".

\section{CONSIDERACIONES FINALES: HACIA LA COMPETENCIA EN LA UNIVERSIDAD DESDE LA BIOLOGIA}

En el transcurso de este ensayo he discutido y explicado el proceso de aprendizaje profesional desde una perspectiva biológica y no informacionista, y he definido competencia profesional desde dicha perspectiva. Considero que este ejercicio resulta en una dinámica saludable para la universidad y la sociedad toda. Como en toda reflexión, la re-explicación de los fenómenos permite reformular los posibles problemas que surgen en torno a ellos y facilitar el hallazgo de soluciones.

Este análisis tiene una serie de implicaciones de importancia para quienes forman parte del proceso de enseñanza superior. Creo que el simple hecho de considerar que no todos los estudiantes son iguales, que no es posible conocer absolutamente las variables que modularán la operatividad de cada uno de ellos y que la labor del docente no ha de ser sólo la de un expositor de descripciones, ya plantea una serie de desafíos metodológicos para el docente que los quiera aceptar. Esto es de radical importancia en estos días de informática y multiplicidad de medios de comunicación. Es, a mi juicio, necesario y urgente rescatar la labor de modelos, tanto de los padres como de los docentes primarios, secundarios y universitarios. Por su plasticidad inherente y su 
capacidad de imitación, el humano pasa gran parte de su ontogenia aprendiendo a ser humano, es decir, aprendiendo las conductas que serán reconocidas como humanas por los otros miembros de su sociedad. Durante otro tanto de su ontogenia, el humano pasa aprendiendo una funcionalidad que le otorgue un papel dentro de la sociedad. Si nos centramos únicamente en una perspectiva informacionista, es posible sostener que un humano puede aprender a ser humano a partir de descripciones, imágenes o sonidos extraídos de un libro, de un computador o de un televisor. No obstante, eso es imposible desde la perspectiva de la Biología del Conocimiento. Un humano sólo aprende a ser humano entre humanos, un hombre aprende a ser hombre entre hombres y mujeres, una mujer entre mujeres y hombres, un niño entre niños, pues sólo la íntegra variedad de estímulos que otorga la sociedad incorpora todos los factores que modulan la ontogenia del humano hacia una coordinación con dicha sociedad. Del mismo modo, un profesional competente es, ante todo, un ser humano, y es mucho más que el dominador de una técnica, ciencia o disciplina, o el generador de un producto determinado. Un profesional competente aprenderá a ser competente entre profesionales competentes.

Dado que la competencia es un atributo otorgado por la sociedad en función de sus necesidades y expectativas, los ambientes que persigan generar profesionales competentes deben estar especial y constantemente atentos a los requerimientos de su sociedad, en todos sus aspectos, antes de exportar modelos basados en necesidades de sociedades externas. Dentro de esto, una gran responsabilidad ha de caer en las organizaciones de profesionales, colegios o sindicatos, que han de esforzarse por determinar las necesidades de la sociedad a la que pertenecen y sirven, con el fin de detectar, discutir y revisar constantemente las expectativas de ésta, y convertirse en el nexo necesario entre la universidad y la sociedad en cuestión. Finalmente, y a raíz del caso de las definiciones y usos teóricos del concepto de competencia, considero que los docentes, intelectuales, teóricos y técnicos en enseñanza y aprendizaje deben estar atentos y ser críticos respecto al sentido de los términos que utilizan, a reconocer si es que tienen o no un significado consensuado, a si son aplicables y a si realmente están siendo definidos atendiendo al sentido y al uso que tienen en el lenguaje propio de cada sociedad. De lo contrario, se corre el riesgo de teorizar ampliamente sobre temas educacionales basándose en términos sin definición o que sencillamente abordan conceptos inexistentes en el lenguaje.

\section{BIBLIOGRAFIA}

Deamer, D., J. Dworkin, S. Sandford, M. Bernstein, L. Allamandola (2002). The first cell membranes. Astrobiology 2: 371-381.

Fleischaker, G. y L. Margulis (1986). Autopoiesis and the origin of bacteria. Adv. Space Res. 6: 53-55.

González, J. y R. Wagenaar (2002). Tuning educational structures in Europe. Informe Final Fase 1. Bilbao: Universidad de Deusto.

LeBoterf, G. (1994). De la compétence: essai sur un attracteur étrange. Paris: Les Éditions d'Organisation.

Letelier, J., G. Marín, J. Mpodozis (2003). Autopoietic and (M,R) systems. J. Theor. Biol. 222: 261-272.

Luhmann, N. (1982). The world society as a social system. Int. J. Gen. Systems 8: 131-138.

Margulis, L. y D. Sagan (1995). What is Life. New York: Simon and Shuster. 
Maturana, H. (1975). The organization of the living: a theory of the living organization. J. ManMachine Studies 7: 313-332.

Maturana, H. (1978). Biology of language: Epistemology of reality. En G. Miller y E. Lenneberg (eds.). Psychology and biology of language and thought (pp. 27-63). New York: Elsevier Science and Technology Books.

Maturana, H. (1982). Aprendizaje o deriva ontogénica. Arch. Biol. Med. Exp. 15: 261-271.

Maturana, H. (1995). Ontología del conversar. En H. Maturana. La realidad: ¿objetiva o construida? Tomo I (pp. 19-36) Rubí (Barcelona): Anthropos.

Maturana, H. y F. Varela (1973). De máquinas y seres vivos: una teoría sobre la organización biológica. Santiago: Editorial Universitaria.

Maturana, H. y F. Varela (1987). The Tree of Knowledge. Boston: Shambhala.

Mavelli, F. y P. Luisi (1996). Autopoietic self-reproducing vesicles: a simplified kinetic model. $J$. Phys. Chem. 100: 16600-16607.

McMullin, B. (2000). Remarks on autocatalysis and autopoiesis. Ann NY. Acad. Sci. 901: 163-174.

Mingers, J. (1995). Self-productions systems: Implications and applications of autopoiesis. New York: Plenum Press.

Nelken, D. y J. Priban (2001). Law's New Boundaries: The consequences of Legal Autopoiesis. Aldershot: Ashgate Publishing.

Real Academia Española (2001). Diccionario de la Lengua Española (p. 604). Madrid: Espasa Calpe.

Ruiz, G. (2005). Closure-embodiment as a valid approach to the understanding of neuropathic pain. Abstracts: 11th World Congress on Pain (p. 81). Seattle: IASP Press.

Savery, J. (2006). Overview of problem-based learning: definitions and distinctions. Interdisc $J$. PBL. 1: 9-20.

Scott, B. (2000). Organizational closure and conceptual coherence. Ann NY. Acad. Sci. 901: 301-310.

Snyder, E. (1999). Is Freud's model of the mind autopoietic? Germanic Review 74: 67-78.

Stewart, J. (2000). From autopoiesis to semantic closure. Ann NY. Acad. Sci. 901: 155-162.

Szostak, J., D. Bartel, P. Luisi (2001). Synthesizing life. Nature 409: 387-390.

Varela, F. (1979). Principles of biological autonomy. New York: Elsevier/North-Holland.

Varela, F., H. Maturana, R. Uribe (1974). Autopoiesis: The organization of living systems, its characterization and a model. BioSystems 5: 187-196.

Zeleny, M. y K. Hufford (1992). The application of autopoiesis in systems analysis: are autopoietic systems also social systems? Int. J. Gen. Systems 21: 145-160. 

Las colaboraciones, pedidos, suscripciones y correspondencia deben ser dirigidas a la Dirección de la revista: Oficina de Publicaciones, Facultad de Filosofía y Humanidades, Universidad Austral de Chile, Casilla 567, Fono/ Fax: 5663 221275, Valdivia, Chile. E-mail: eped@uach.cl

Las suscripciones incluyen los gastos de envío:

Chile: \$2.000. Otros países: US\$ 10.

El canje debe ser enviado a Biblioteca Central, Universidad Austral de Chile, Correo 2, Valdivia 